\title{
Estimativa dinâmica da pressão alveolar pulmonar em diferentes modos ventilatórios controlados
}

\author{
Glauco Cabral Marinho Plens ${ }^{1}$, Eduardo Leite Vieira Costa ${ }^{2}$, Mauro Roberto Tucci², Carlos \\ Roberto Ribeiro de Carvalho ${ }^{2}$
}

Plens GCM, Costa ELV, Tucci MR, Carvalho CRR. Estimativa dinâmica da pressão alveolar pulmonar em diferentes modos ventilatórios controlados. Rev Med (São Paulo). 2015 jan,-mar.;94(1):71-2.

\section{INTRODUÇÃO}

O conhecimento da quantidade de colapso alveolar pulmonar é importante no manejo de pacientes com síndrome do desconforto respiratório agudo (SDRA). Atualmente, o padrão-ouro dessa medida é a tomografia computadorizada de raios-X, a qual requer o transporte do paciente para a radiologia e um pós-processamento laborioso e lento. Recentemente foi descrito um método capaz de estimar em tempo real o colapso pulmonar em pacientes em ventilação mecânica invasiva com base na Tomografia por Impedância Elétrica (TIE) e na mecânica pulmonar. Esse método apresenta como limitação a necessidade de ajustes ventilatórios que proporcionem pausas inspiratória e expiratória. Existem alguns métodos descritos, como o da regressão linear múltipla (MLR) ou do isovolume, que permitem a avaliação da mecânica respiratória em condições dinâmicas. Essa possibilidade de monitorizar a mecânica do sistema respiratório sem a necessidade de padrão ventilatório específico ou períodos de pausa permitiria a estimativa do colapso pulmonar através da TIE em qualquer paciente sob ventilação mecânica controlada de forma robusta e pouco dependente do operador.

\section{OBJETIVO}

Avaliar, comparados à mecânica respiratória estática, a acurácia de dois métodos de estimativa da mecânica respiratória que prescindem de ajustes ventilatórios específicos: método da MLR e método do isovolume.

\section{METODOLOGIA}

$\mathrm{O}$ estudo foi realizado em um simulador mecânico de complacência constante e igual a $72,9 \mathrm{~mL} / \mathrm{cmH}_{2} \mathrm{O}$. Definimos como inadequadas estimativas de complacência que tivessem um erro $>10 \%$ desse valor nominal. $\mathrm{O}$ sistema de medidas era composto de um transdutor de pressão e um sensor de fluxo adaptados no circuito entre o ventilador mecânico e o simulador, cuja pressão interna (correspondente à pressão alveolar) foi medida utilizando outro transdutor de pressão. Medidas de pressão alveolar e complacência foram obtidas ventilando o simulador nos modos ventilatórios pressão controlada e volume controlado, com diversos ajustes, incluindo pausa ao final da inspiração e com uso de duas resistências de vias aéreas

\footnotetext{
$2^{\circ}$ lugar Prêmio Painéis - Área Básica no 33 COMU - Congresso Médico Universitário da FMUSP, SP, 31 de out. a 02 de nov. de 2014. 1. Acadêmico de Medicina da Faculdade de Medicina da Universidade de São Paulo.

2. Orientadores, Faculdade de Medicina da Universidade de São Paulo.
} 
produzidas pela adição no circuito de resistores lineares. As complacências estimadas pelo método de MLR foram obtidas a partir de ciclos respiratórios inteiros ou evitando as transições entre inspiração e expiração, ou seja, somente com regiões de interesse (ROI).

\section{RESULTADOS}

Foram adequadas 59,7\% das medidas estimadas pelo método da MLR utilizando ciclos inteiros, $50,8 \%$ das obtidas pela MLR com ROI e 46,8\% das estimadas pelo método do isovolume, de um total de 62 medidas. A adição de um componente de fluxo quadrático ao modelo da MLR aumentou para $70,4 \%$ o percentual de medidas adequadas a partir de ciclos inteiros e para $69,8 \%$ utilizando ROI. A pressão alveolar estimada pela MLR correlacionou-se bem com a pressão interna no simulador $\left(\mathrm{R}^{2}=0,97, \mathrm{p}<0,001\right)$.

\section{DISCUSSÃO}

Entre os métodos testados, a MLR fez estimativas adequadas da complacência e da pressão alveolar na maioria dos casos. Entretanto, mostrou-se importante inserir um componente de fluxo quadrático na equação do movimento para aprimorar a acurácia do método, indicando que a existência de fluxo turbulento prejudica a estimativa. Também foi verificado que evitar transições entre inspiração e expiração, habitualmente ruidosas, não melhora a acurácia da MLR. 